\title{
UTILISATION D'UN TEST DE RANDOMISATION POUR DÉTECTER L'EFFET DE REJETS POLLUANTS DANS UN COURS D'EAU : APPLICATION À L'IMPACT D'EFFLUENTS DE PISCICULTURES SUR LA PRODUCTION DE JUVÉNILES DE SAUMON ATLANTIQUE.
}

\author{
E. PRÉVOST
}

Laboratoire d'Écologie Aquatique, INRA, 65 rue de St Brieuc, 35042 Rennes Cedex, France.

\section{RÉSUMÉ}

La mise en évidence de l'impact de la pollution à l'échelle des populations animales et végétales d'un cours d'eau doit reposer sur une analyse comparative entre une situation de référence (i.e. " non polluée ») et une situation soumise à la pollution. A l'occasion de l'étude d'un cas concret, l'impact sur la production de juvéniles de saumon atlantique (Salmo salar) des pollutions ponctuelles engendrées par les effluents de piscicultures intensives sur un petit fleuve côtier breton (le Scorff), on propose une approche méthodologique qui s'appuie sur des observations quantitatives distribuées le long du cours d'eau concernant la population étudiée et sur une définition claire de critères objectifs d'identification d'une station perturbée par la pollution. Une démarche pas à pas ad hoc est élaborée, au cours de laquelle on utilise à chaque pas une procédure de test de randomisation originale, adaptée au traitement de séries d'observations d'abondance ordonnées dans l'espace et soumise à une source de variation ponctuelle par rapport à laquelle on souhaite effectuer une comparaison.

L'abondance des juvéniles de saumon de l'année a été suivie sur le Scorff par pêche électrique de 1993 à 1998 sur un réseau d'une quarantaine de stations échantillonnées chaque automne. Sur la base du jeu de données ainsi collecté, l'application de la méthodologie proposée permet de mettre en évidence que les deux piscicultures de truite arc-en-ciel (Oncorhynchus mykiss) installées sur le cours principal, produisant chacune plusieurs centaines de tonnes par an, occasionnent des réductions significatives d'abondance en tacons $0_{+}$, sur des distances de plusieurs kilomètres à l'aval des points de rejets de leurs effluents d'élevage.

Ces résultats sont discutés, aussi bien par rapport à l'approche méthodologique employée que du point de vue des perturbations écologiques engendrées par les installations de pisciculture intensive. 


\title{
USE OF A RANDOMISATION TEST \\ FOR DETECTING THE EFFECT OF POLLUTING DISCHARGES IN A STREAM : APPLICATION TO THE IMPACT OF FISH FARM EFFLUENTS ON THE PRODUCTION OF ATLANTIC SALMON JUVENILES.
}

\begin{abstract}
The evidence of the impact of pollution on animal or plant populations in a river must be based on a comparative analysis between a status of reference (i.e. non-polluted) and a situation submitted to pollution. While presenting a case study, the impact of the effluents of intensive aquaculture facilities on the production of juvenile Atlantic salmon (Salmo salar) in a short coastal stream of Brittany (Scorff R.), a methodological approach is proposed which rests on quantitative observations spread out along the river course and a clear definition of objective criteria of identification of stations perturbed by the pollution. An ad hoc stepwise process is worked out, which uses at each step an original procedure of randomisation test, suited to the analysis of observation series of abundance ordered spatially and submitted to a point source of variation in relation to which a comparison is intended.
\end{abstract}

The abundance of the young of the year salmon has been surveyed on the Scorfl by electrofishing from 1993 to 1998 on forty stations sampled every autumn. Based on the data set thus collected, the implementation of the proposed methodology allows to demonstrate that the two rainbow trout (Oncorhynchus mykiss) farms installed on the mainstream, each producing several hundred tons per year, occasion significant reductions in $0+$ parr abundance, over distances of several kilometres downstream from the throwing point of the effluents of the rearing facilities.

These results are discussed as well regarding the methodological approach employed as in connection with the ecological perturbations caused by the intensive fish farms.

\section{INTRODUCTION}

Les activités humaines qui se développent le long des cours d'eau modifient les conditions de vie des espèces aquatiques, qu'elles soient animales ou végétales. Ces modifications environnementales correspondent le plus souvent à une pollution des rivières, au sens défini par RAMADE (1993, p. 513). La mise en évidence de l'impact de la pollution à l'échelle des populations animales et végétales d'un cours d'eau doit reposer sur une analyse comparative entre une situation de référence (i.e. "non polluée ") et une situation soumise à la pollution. Dans le cas d'une pollution ponctuelle, i.e. dont la source est bien localisée dans l'espace, on pourra comparer, à l'intérieur d'un même réseau hydrographique, des sites affectés par cette pollution avec d'autres similaires qui ne le sont pas. Dans une analyse comparative de ce type, le statut " non pollué » est á prendre dans un sens relatif, en particulier quand on s'intéresse à des écosystèmes fortement anthropisés. En effet, on cherche à mettre en évidence l'effet marginal d'une source de pollution ponctuelle, par rapport à un état de référence qui peut déjà être affecté par les activités humaines.

Dans la pratique, une telle approche nécessite de disposer de données sur les populations étudiées couvrant à la fois des zones polluées et non polluées, ainsi que d'une méthode de comparaison. RAGO (1993) propose un outil statistique de comparaison original. II s'agit d'une procédure de test de randomisation, ne nécessitant que peu d'hypothèses, adaptée au traitement conjoint de séries multiples d'observations 
d'abondance par dénombrement ordonnées dans l'espace ou dans le temps et soumises à une source de variation ponctuelle par rapport à laquelle on veut faire une comparaison ("amont-aval " ou « avant-après " par exemple).

Dans le présent article, on se propose d'utiliser cet outil pour l'étude d'un cas concret, l'impact sur la production de juvéniles de saumon atlantique (Salmo salar) des pollutions ponctuelles engendrées par des installations de piscicultures intensives sur un cours d'eau breton, le Scorff. Les effluents d'élevage aquacoles constituent des sources de pollution (FAURÉ, 1977 ; BOURGET-RIVOALLAN, 1982 ; KENDRA, 1991 ; OBERDORFF et PORCHER, 1994 ; DANIEL et HAURY, 1995) car ils sont, entre autres, chargés en matières en suspension (résidus d'aliments et fèces), enrichis en substances azotées (excrétion d'ammoniaque par les poissons) et contiennent des résidus de produits de traitements utilisés pour contrôler l'état sanitaire du cheptel d'élevage (métaux lourds, antibiotiques). Ils modifient donc la qualité de l'eau et du lit (dépôts de boues) de la rivière en aval. Le Scorff, petit fleuve côtier qui se jette dans la rade de Lorient (Morbihan), se prête particulièrement bien à l'étude de l'impact des pollutions ponctuelles occasionnées par les piscicultures intensives sur la production naturelle de juvéniles de saumon. En effet, on trouve sur son cours principal deux importantes installations d'élevage de truite arc-en-ciel (Oncorhynchus mykiss) et l'abondance des tacons y est suivie annuellement par pêche électrique sur un réseau d'une quarantaine de stations depuis 1993.

L'objet de ce travail est double. Il est méthodologique, avec le souci de proposer et d'illustrer une démarche pouvant être extrapolée à d'autres cas. Cette démarche présente des éléments novateurs à deux niveaux, celui de la quantification de l'abondance des populations in situ et celui de l'analyse statistique des informations collectées. Au-delà de cet aspect méthodologique, l'objet est aussi de traiter d'une question spécifique, l'impact de la pollution des piscicultures intensives sur les populations de saumon en Bretagne. L'intérêt particulier de cette question est sous-tendu par la conjonction de plusieurs éléments :

- le saumon atlantique est une espèce réputée sensible à la dégradation des écosystèmes aquatiques sous l'influence de l'homme. Ainsi, le nombre de cours d'eau qu'elle fréquente le long des côtes de l'Atlantique nord et en particulier en France a subi une restriction significative depuis deux siècles (THIBAULT, 1987). La valeur patrimoniale de cette espèce, symbolique et emblématique à bien des égards (THIBAULT, 1996), s'en est trouvée renforcée.

- la Bretagne est à la fois le principal refuge du saumon atlantique en France, avec plus de 20 fleuves côtiers soutenant encore des populations naturelles, et la première région française de production de truite arc-en-ciel en pisciculture intensive.

\section{MATÉRIEL ET MÉTHODES}

\section{Site d'étude}

Long de $75 \mathrm{~km}$ (dont $10 \mathrm{~km}$ d'estuaire), le Scorff (Figure 1) draine une surface de bassin versant de $480 \mathrm{~km}^{2}$. Son débit moyen annuel dans sa partie basse est d'environ $5 \mathrm{~m}^{3 / \mathrm{s}}$. Il coule sur un substrat essentiellement granitique mais traverse deux bandes schisteuses engendrant deux ruptures de pente sur son cours principal. II est colonisé par une quinzaine d'espèces de poissons, dont quatre sont des migrateurs amphihalins: la lamproie marine (Petromyzon marinus), l'anguille européenne (Anguilla anguilla), la truite commune (Salmo trutta) et le saumon atlantique (Salmo salar). II fait donc partie de la vingtaine de cours d'eau bretons à saumon dont il est un élément bien représentatif. Le saumon est présent sur son cours principal, depuis la limite de balancement des marées à 
Pont-Scorff, jusqu'à Guémené sur Scorff (50 km en amont) où la remontée des adultes est bloquée par un obstacle infranchissable. On le trouve également sur quelques petits affluents de sa partie basse, mais leur contribution à la production des juvéniles est marginale (CLAUDE. 1996).
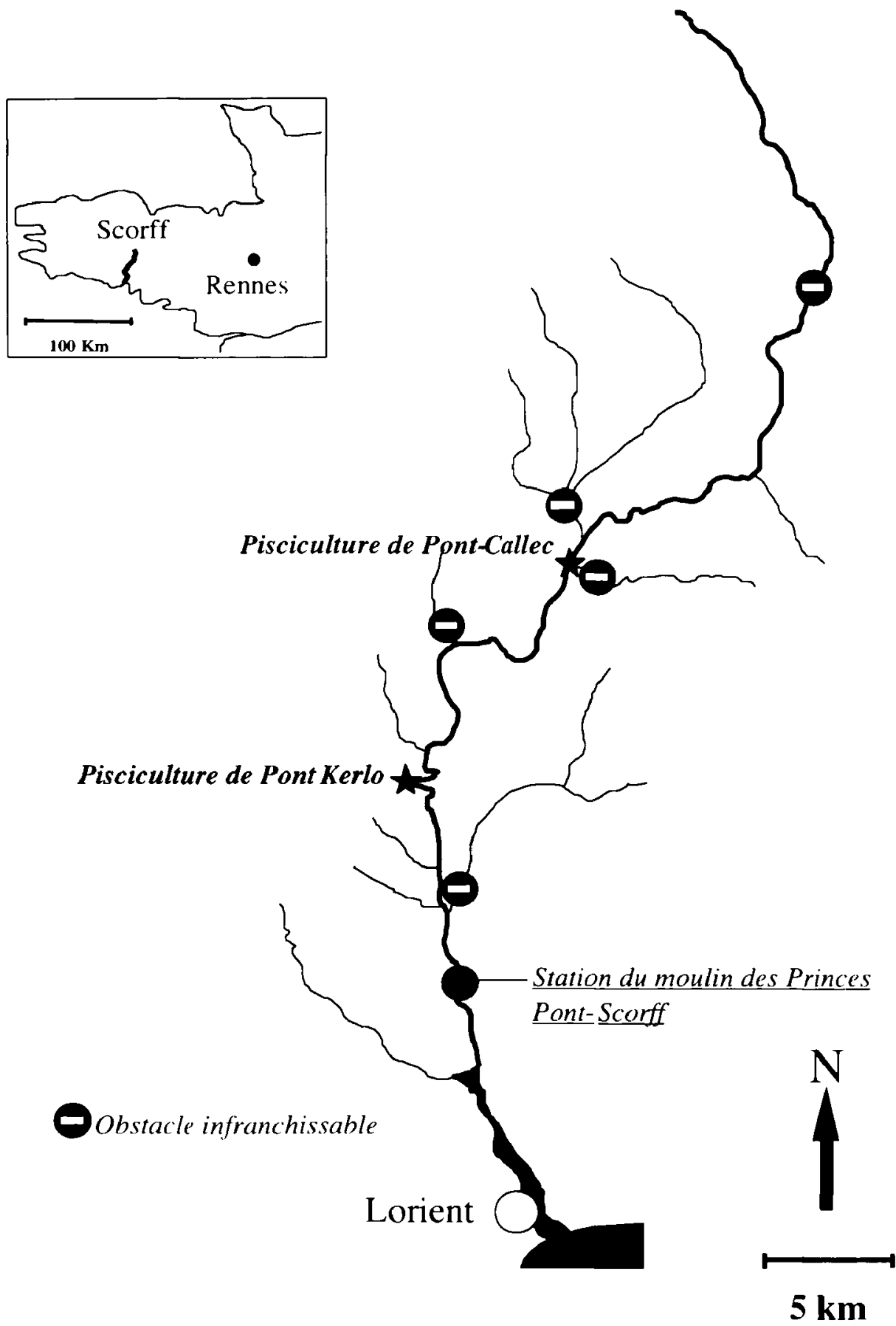

\section{Figure 1}

Le réseau hydrographique du Scorff: localisation des deux piscicultures du cours principal.

\section{Figure 1}

The Scorff R. system : localisation of the two fish farms on the mainstream. 


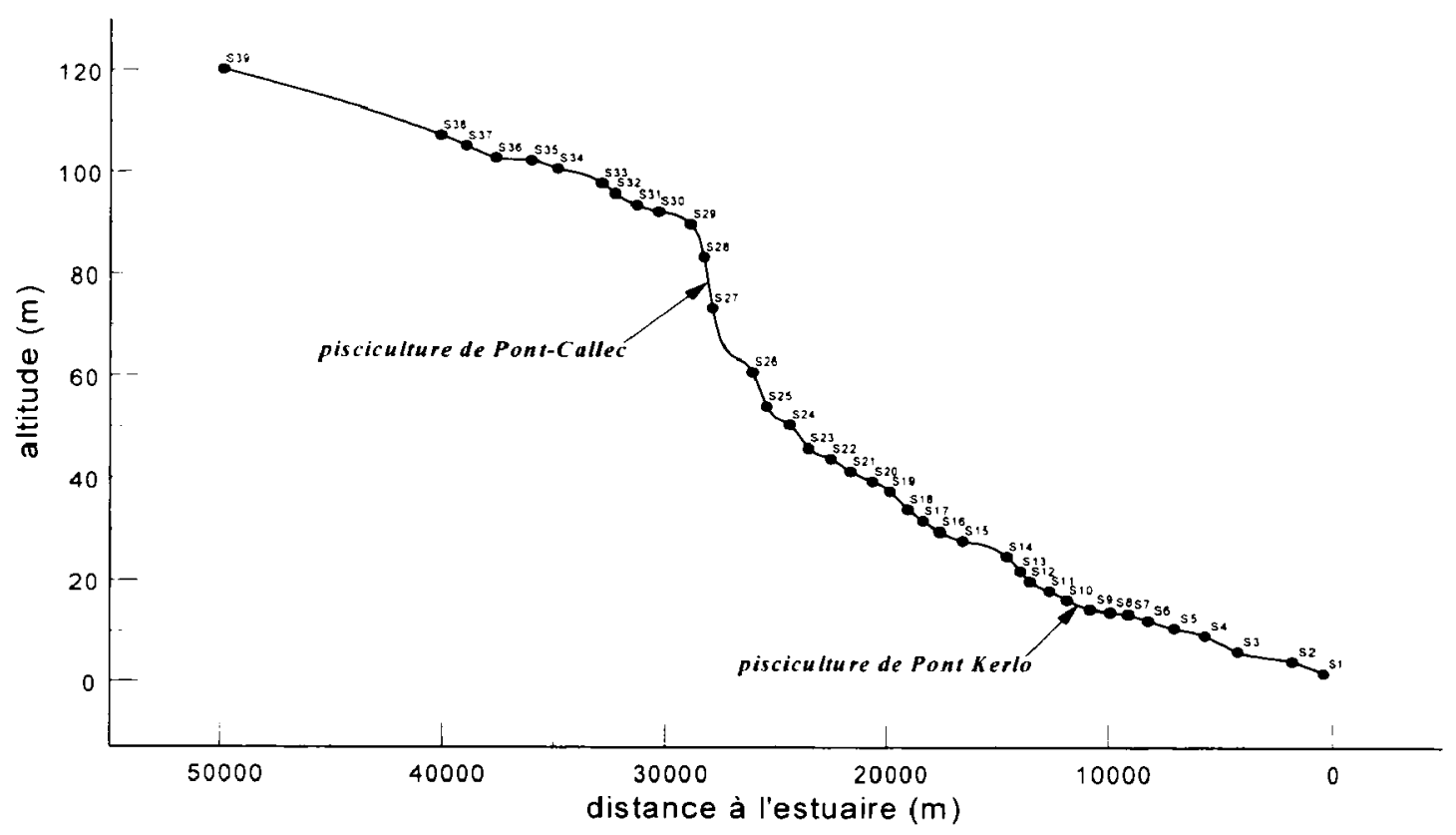

Figure 2

Profil en long du cours principal du Scorff : localisation des stations d'échantillonnage des juvéniles de saumon atlantique et des deux piscicultures.

\section{Figure 2}

Longitudinal profile of the Scorff R. mainstream : localisation of the juvenile Atlantic salmon sampling stations and of the two fish farms.

Deux piscicultures intensives de truite arc-en-ciel sont installées sur le cours principal du Scorff (Figure 1). On ne dispose pas de données fiables permettant de quantifier précisément leur production annuelle, mais on peut grossièrement l'évaluer à plusieurs centaines de tonnes chacune. Ces deux installations sont situées dans des contextes assez différents (CLAUDE, 1996). Celle de Pont Kerlo, la plus en aval $(11 \mathrm{~km}$ de la limite de remontée de la marée), se trouve au-dessus d'une zone à faible pente (Figure 2) où prédomine les faciès d'écoulement laminaires et relativement lents. Celle de Pont-Callec ( $28 \mathrm{~km}$ de la limite de remontée de la marée) est à l'amont de la partie la plus pentue du Scorff (Figure 2), riche en faciès d'écoulement turbulent et à fortes vitesses de courant. Chacune des deux piscicultures dérive grâce à un barrage une partie du débit du Scorff pour ses besoins propres, puis rejette l'eau ayant transité au travers des bassins d'élevage immédiatement en aval. Les barrages ne sont pas des barrières infranchissables pour la remontée des saumons adultes, mais ils constituent cependant des obstacles à la libre circulation des poissons.

\section{Données d'abondance de la population de juvéniles de saumon de l'année}

La population de juvéniles de saumon atlantique du Scorff est échantillonnée chaque automne (dernière semaine de septembre) depuis 1993 sur la base d'un ensemble d'une cinquantaine de stations réparties sur l'ensemble du réseau hydrographique. Trente-neuf d'entre elles sont situées sur la zone du cours principal colonisée par le saumon (Figure 2). Elles correspondent aux faciès d'écoulement préférentiellement utilisés par les tacons en automne, c'est-à-dire peu profonds, à écoulement rapide et à fond grossier. Ces faciès sont généralement turbulents en surface et correspondent aux catégories " radiers " et "rapides " définies par BAGLINIĖRE et CHAMPIGNEULLE (1986). Les stations ont été choisies en cherchant un compromis entre un espacement 
régulier entre elles et un effort d'échantillonnage plus intense sur les zones à forte densité d'habitats favorables aux juvéniles. Compte tenu de la position des deux piscicultures sur le cours principal, aussi bien du point de vue de la distance à l'estuaire que par rapport à la distribution longitudinale des faciès d'écoulement favorables aux tacons (Figure 2), on aboutit à la répartition des stations suivante: 9 en aval de l'installation de Pont Kerlo, 18 entre les deux piscicultures et 12 en amont de celle de Pont-Callec.

Sur chaque station des indicateurs d'abondance en tacons de l'année $(0+)$ de type " capture par unité d'effort » sont collectés annuellement selon le protocole de pêche électrique standardisé défini par PRÉVOST et BAGLINIĖRE (1995). Ces indicateurs d'abondance, exprimés en nombre de poissons capturés par cinq minutes de pêche (unité notée ind $/ 5 \mathrm{mn}$ par la suite), sont proportionnels à des densités de populations estimées par la méthode des enlèvements successifs (PRÉVOST et NIHOUARN, 1999). Tous les tacons capturés lors des opérations de pêche électrique sont mesurés (longueur fourche, $\mathrm{mm}$ ). Ils appartiennent quasi exclusivement à deux classes d'âge : $0+$ et $1+$ (les individus d'âge supérieur sont exceptionnels). Les tacons de l'année peuvent être séparés de ceux plus âgés par une simple observation de la distribution des tailles, sauf dans certains cas douteux où un prélèvement d'écailles est réalisé pour la détermination de l'âge.

\section{Traitement statistique des données}

Les données utilisées portent uniquement sur les tacons de l'année car seule cette classe d'âge reflète directement l'abondance d'une cohorte (année de naissance) au cours de sa phase juvénile en rivière. En effet, la migration des jeunes vers la mer se faisant majoritairement à l'âge d'un an (PRÉVOST, 1998), les tacons plus âgés proviennent du reliquat d'individus n'ayant pas encore quitté leur rivière natale.

L'identification des stations influencées négativement par la pollution des piscicultures est menée globalement sur les six années d'étude (1993-1998). Elle repose sur les postulats suivants:

(I) l'influence d'une pisciculture débute à partir du point de rejet des effluents et se propage de façon continue et uniquement vers l'aval ;

(II) compte tenu du pouvoir auto-épurateur de la rivière, l'influence d'une pisciculture est négligeable au-delà d'un certain point en aval ;

(III) l'impact négatif des rejets polluants d'une pisciculture sur la production de juvéniles de saumon est associé à des réductions d'abondance en tacons de l'année.

Une des conséquences du postulat (I) est d'interdire l'identification comme perturbée de toute station qui serait précédée à l'amont par au moins une station non perturbée. On cherche ainsi à éviter d'attribuer à l'influence d'une pisciculture des faibles abondances locales en tacons qui pourraient être dues à d'autres facteurs (tels des particularités du milieu au voisinage de certaines stations entraînant un déficit en habitat de reproduction).

Conformément aux postulats qui viennent d'être énoncés, on considérera qu'une station est perturbée par une pisciculture si elle remplit simultanément les trois conditions suivantes:

(i) elle est située à l'aval de l'installation aquacole considérée ;

(ii) elle appartient à un continuum de stations perturbées débutant à la première station à l'aval immédiat de cette dernière ; 
(iii) globalement sur les six années d'étude, l'abondance en tacons $0+y$ est significativement inférieure à celle d'une base de référence regroupant des stations non perturbées circonvoisines.

Pour mener de front la vérification de ces trois critères, une démarche pas à pas ad hoc a été utilisée. A chaque pas, une station est comparée avec une base de référence, constituée de stations non perturbées situées en amont de la pisciculture concernée ainsi qu'en aval de la station considérée. On passe d'un pas à l'autre en progressant d'une station vers l'amont, la base de référence étant mise à jour en fonction du résultat obtenu au pas précédent. Si ce dernier a révélé un niveau d'abondance significativement déprimé, la base de référence reste inchangée. Dans le cas contraire, la dernière station examinée est ajoutée à la base de référence, de même qu'éventuellement les stations directement en aval pour lesquelles des abondances significativement réduites auraient été révélées lors des pas antérieurs. Ainsi, à chaque nouveau pas, la base de référence est augmentée de toute station identifiée comme non-perturbée au pas précédent. En effet, conformément au postulat (l) et à la condition (ii), toute station pour laquelle une réduction significative d'abondance est détectée est considérée comme non perturbée par la pisciculture concernée dès lors qu'une autre station située en amont ne montre pas une telle diminution. La comparaison d'une station par rapport à sa base de référence réalisée à chaque pas est faite à partir des données d'abondance collectées au cours des six années d'étude et selon un critère objectif. La procédure statistique de test de randomisation proposée par RAGO (1993) a été employée car elle constitue une voie d'approche bien adaptée à notre cas, à la fois du point de vue des données traitées et de la question posée.

Cette procédure s'intéresse à l'effet simultané d'une source de variation ponctuelle dans le temps (par exemple, une modification de la réglementation) ou dans l'espace (par exemple, un rejet polluant) sur un ensemble de séries d'observations d'abondance ordonnées dans le temps ou dans l'espace. La méthode est décrite précisément par RAGO (1993) avec des indications sur la puissance du test. Pour en saisir le principe, on retiendra qu'elle suppose que l'on dispose d'un jeu de données se présentant sous la forme d'un tableau rectangulaire, constitué de $K$ séries (colonnes) comprenant chacune $m+n$ observations (lignes). Ce tableau est scindé en un sous-ensemble de référence, constitué des $\mathrm{K} \times \mathrm{m}$ premières observations, et un sous-ensemble ayant subi l'influence de la source de variation ponctuelle dont on souhaite détecter l'effet éventuel, comprenant les $K \times n$ observations restantes. On calcule alors le rapport $R_{0}$ entre la somme des abondances moyennes par série pour le sous-ensemble soumis à l'influence de la source de variation ponctuelle et la somme des abondances moyennes par série pour le sous-ensemble de référence, soit :

$$
R_{0}=\frac{\left.\sum_{i=1}^{K} \mid \sum_{j=1}^{n} A_{i j} / n\right)}{\sum_{i=1}^{K}\left(\sum_{j=1}^{m} A_{i j /} /\left.m\right|_{\text {ref }}\right.}
$$

où $A_{i j}$ est l'abondance de tacons de l'année sur la station j pour l'année i.

Ce rapport vaut 1 si la source de variation n'a pas d'effet sur l'abondance, est supérieur à 1 si la source de variation a entraîné une augmentation d'abondance et est inférieur à 1 si la source de variation a conduit à une diminution d'abondance. Une approximation de la distribution d'échantillonnage de $R_{0}$, sous l'hypothèse nulle que les observations sont ordonnées au hasard dans chaque série, est obtenue par des permutations aléatoires ("randomisation ") des données. Le positionnement de la valeur 
de $R_{0}$ observée dans la distribution ainsi obtenue permet d'estimer la probabilité d'observer une valeur supérieure (ou inférieure) à $R_{t,}$ sous l'hypothèse nulle. En fixant une valeur seuil à cette probabilité (niveau de signification), on obtient une procédure de test d'hypothèse. Par rapport aux méthodes usuelles de comparaison de moyennes (test-t par exemple), la procédure proposée par RAGO (1993) permet de s'affranchir des contraintes concernant l'indépendance des observations et l'égalité des variances. En outre, elle reste applicable même quand l'un des sous-ensembles comporte seulement une observation par série. Ceci est particulièrement intéressant dans notre cas où, chaque station potentiellement perturbée est comparée individuellement $(n=1)$ par rapport à une base de référence en s'appuyant sur six séries d'observations annuelles $(K=6)$. La mise en cuvre pratique de cette procédure est menée au moyen du programme informatique fourni sur simple demande par l'auteur (RAGO, 1993). Dans le présent travail, les tests ont été réalisés au seuil de $10 \%$, pour augmenter la puissance de la procédure par rapport au seuil classique de $5 \%$, et 5000 permutations aléatoires des données ont été effectuées pour chaque test.

La pisciculture de Pont-Callec est examinée en premier, car l'identification de stations non perturbées en amont direct de l'installation de Pont Kerlo (mais en aval de celle de Pont-Callec; Figure 1) est un préalable indispensable avant l'analyse de l'impact de cette dernière. Bien que suivant exactement le même principe général énoncé précédemment, le processus pas à pas de traitement des données est adapté dans le détail au cas particulier de chacune des deux piscicultures. II se déroule précisément comme suit :

\section{- Pisciculture de Pont-Callec}

- Pas 1: les 10 premières stations en amont de la pisciculture de Pont-Callec (stations 28 à 37, numérotation progressant vers l'amont ; Figure 2) sont prises comme base de référence initiale, soit six séries annuelles de 10 mesures d'abondances (60 données élémentaires). Les deux stations les plus amont (38 et 39) sont exclues de la base de référence, considérant que les faibles abondances fréquemment observées sur ces stations (Figure 3) peuvent être dues à leur position extrême sur la zone du cours principal du Scorff colonisée par le saumon. On compare à cette base la première station à l'amont immédiat de la pisciculture de Pont Kerlo (station 10), soit six séries d'une mesure d'abondance (six données élémentaires), en utilisant la procédure de test de RAGO (1993). On débute le processus pas à pas au-dessus de la pisciculture de Pont Kerlo pour éviter toute confusion entre l'effet des deux installations. Si la station 10 ne présente pas des abondances significativement plus faibles au seuil de $10 \%$ que sur la zone de référence, elle est considérée comme non perturbée et elle est incluse dans la base de référence pour le pas suivant.

- Pas $i$ : la base de référence est constituée des 10 stations à l'amont de la pisciculture de Pont-Callec et des $j(j<i)$ stations $(10$ à $10+j-1)$ identifiées comme non perturbées au pas $\mathrm{i}-1$. La station 10+i-1 est comparée à cette base de référence. Si le test est significatif au seuil de $10 \%$, la base de référence restera inchangée au pas suivant. Si le test n'est pas significatif, alors la base de référence comprendra au pas ultérieur l'ensemble des stations 28 à 37 et 10 à $10+i-1$.

- Dernier pas : on compare la station 27, à l'aval immédiat de la pisciculture de Pont-Callec avec la base de référence déduite du pas précédent.

\section{- Pisciculture de Pont Kerlo}

Le cas de la pisciculture de Pont Kerlo est traité de la même façon, à ceci près que l'on débute la procédure pas à pas à la première station échantillonnée à l'aval du cours 
principal du Scorff, avec pour base de référence initiale l'ensemble des stations situées à son amont direct et qui n'ont pas été identifiées comme perturbées par la pisciculture de Pont-Callec. Les stations 1 et 2 (Figure 3) présentent des abondances en juvéniles de saumon de l'année plus faibles que les deux stations suivantes à l'amont, ce qui pourrait être dû à leur position extrême sur la zone du cours principal du Scorff colonisée par le saumon. L'effectif de stations échantillonnées annuellement en aval de la pisciculture de Pont Kerlo étant peu important (9), il a été choisi de conserver les deux premières stations pour le traitement des données, afin de ne pas trop réduire le nombre de stations contribuant à l'analyse située en aval de cette installation.

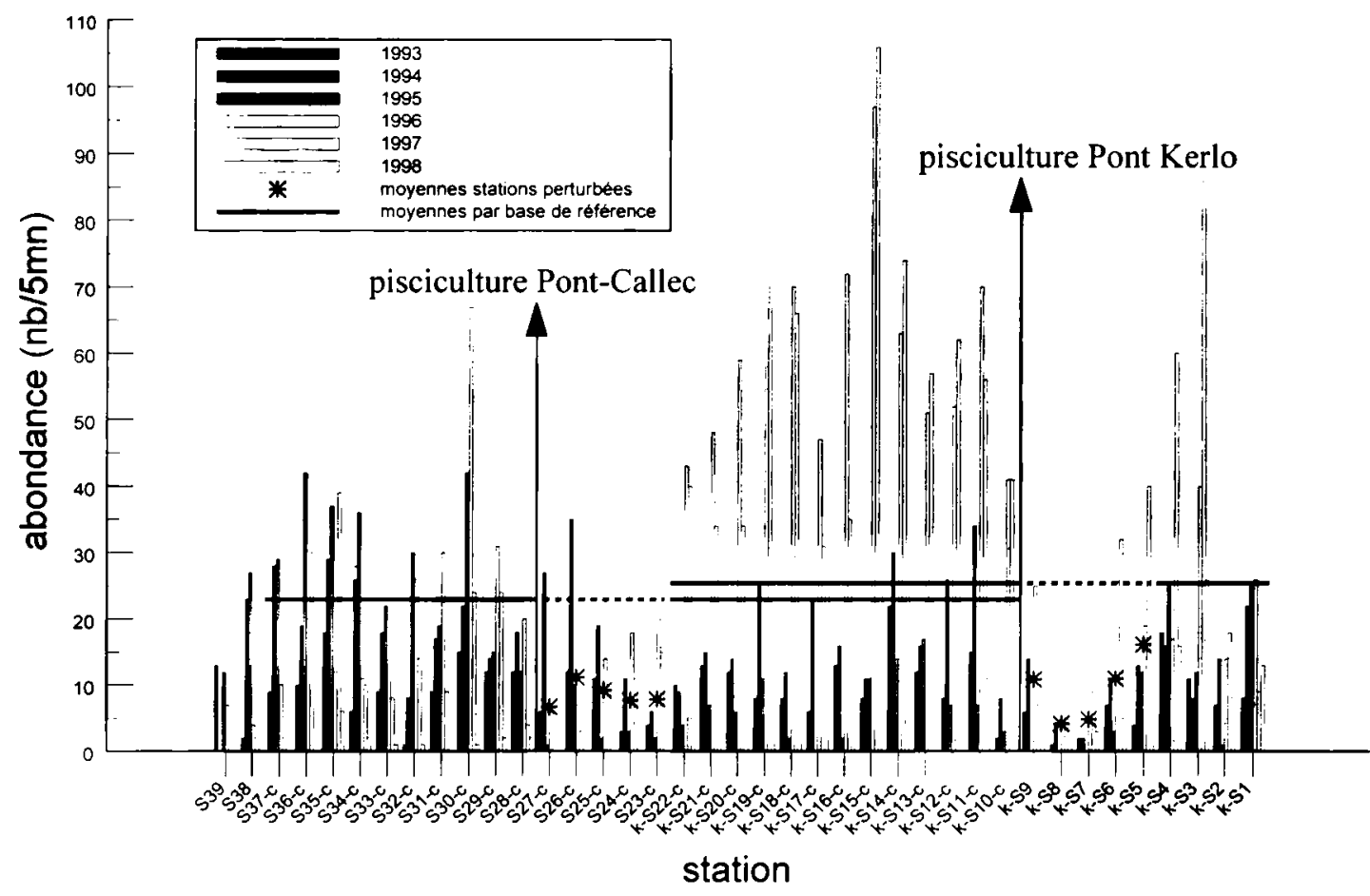

Figure 3

Indicateurs d'abondance en tacons 0+ automnaux par station et par année le long du cours principal du Scorff. Les stations constituant la base de référence par rapport à laquelle ont été mises en évidence les stations perturbées sont suffixées avec un " $c$ " dans le cas de la pisciculture de Pont-Callec et sont préfixées avec un “ $k$ » pour l'installation de Pont Kerlo.

\section{Figure 3}

$0+$ parr indices of abundance by station and by year along the mainstream of the Scorff $R$. The stations which constitute the base of reference in relation to which the perturbed stations have been identified have a " $c$ " suffix added for the Pont-Callec fish farm and have $a$ " $k$ » prefix added for the Pont Kerlo facility.

La démarche utilisée est conforme aux postulats de départ et aux critères d'identification des stations perturbées qui en découlent. A la fin du processus pas à pas on obtient (sous réserve de tests significatifs) pour chaque pisciculture, une succession continue de stations depuis leur aval immédiat qui présentent, globalement sur les six années d'étude, des abondances en tacons d'âge 0+ significativement inférieures à celles observées sur une base de référence. Par sa progression vers l'amont, la démarche pas à pas permet de compléter à chaque pas la base de référence par les stations aval qui n'ont pas été déterminées jusqu'alors comme perturbées, tout en assurant que l'ensemble des 
stations perturbées auront toutes été identifiées par comparaison avec une même base de référence. La base de référence change cependant d'une pisciculture à l'autre, dans le souci de l'adapter en fonction des conditions de milieu rencontrées dans le voisinage de chacune des installations.

\section{RÉSULTATS}

\section{Pisciculture de Pont-Callec}

Au cours du déroulement de la procédure pas à pas, aucun test significatif n'est obtenu avant la station 23 (Figure 4). Par la suite les tests sont significatifs à chaque pas, conduisant à identifier à l'aval direct de la pisciculture de Pont-Callec cinq stations perturbées (23 à 27), réparties sur une distance d'environ $5 \mathrm{~km}$ depuis le point de rejet des effluents polluants. Ces cinq stations ont toutes été comparées par rapport à une même base de référence constituée de 23 stations (Figure 3), 10 à l'amont de l'installation (stations 28 à 37) et 13 à l'aval (stations 10 à 22 pour lesquelles les tests sont non significatifs). Les stations de référence amont présentent en moyenne sur la période 1993-98 des abondances en tacons de l'année plus faibles $(16,9 \mathrm{ind} / 5 \mathrm{mn})$ que les 13 stations aval $(27,5 \mathrm{ind} / 5 \mathrm{mn})$. Quelle que soit la disparité amont-aval dans la base de référence on observe, globalement sur les six années d'étude, des abondances sensiblement réduites sur les cinq stations perturbées (moyenne : $8,5 \mathrm{ind} / 5 \mathrm{mn}$ ). Le rapport $R_{0}$ est toujours nettement inférieur à 1 : de 0,48 à 0,29 suivant la station, pour des probabilités associées inférieures à $3,2 \%$ (Figure 4). A la suite de ces cinq stations perturbées, on trouve trois stations (22 à 20) pour lesquelles $R_{0}$ est encore inférieur à 1 mais dont les tests sont non significatifs. Ces trois stations réparties sur un peu moins de $3 \mathrm{~km}$ paraissent constituer une transition, avant un ensemble de 10 stations du cours intermédiaire du Scorff en amont de la pisciculture de Pont Kerlo, sur lesquelles, si l'on en juge par les abondances élevées qui y sont observées (Figure 3), la production de juvéniles de saumon de l'année ne semble pas affectée par la pisciculture de Pont-Callec.

\section{Pisciculture de Pont Kerlo}

Globalement sur les six années d'étude, les abondances en tacons 0+ sur les deux premières stations ( 1 et 2 ) introduites dans la procédure pas à pas sont significativement inférieures à celles observées sur la base de référence constituée des 13 stations situées à l'amont direct de la pisciculture de Pont Kerlo et qui n'ont pas été identifiées comme perturbées par l'installation de Pont-Callec (Figures 3 et 4). Néanmoins, puisque au pas 3 un test non significatif est obtenu, les deux premières stations sont réintégrées dans la base de référence pour le pas suivant. A compter de la station 5 , les tests sont significatifs à chaque pas, conduisant à identifier à l'aval direct de la pisciculture de Pont Kerlo cinq stations perturbées (5 à 9) réparties sur une distance d'environ $4 \mathrm{~km}$ depuis le point de rejet des effluents polluants. Ces 5 stations ont toutes été comparées avec une même base de référence constituée de 17 stations (Figure 3), 13 à l'amont de l'installation (stations 10 à 22) et 4 à l'aval (stations 1 à 4). Les stations de référence amont présentent en moyenne sur la période 1993-98 des abondances en tacons de l'année plus fortes (27,5 ind $/ 5 \mathrm{mn}$ ) que les 4 stations aval $(19,9 \mathrm{ind} / 5 \mathrm{mn})$. Quelle que soit la disparité amont-aval dans la base de référence on observe, globalement sur les six années d'étude, des abondances sensiblement réduites sur les cinq stations perturbées (moyenne: $9,4 \mathrm{ind} / 5 \mathrm{mn}$ ). Pour la dernière station perturbée en aval de la pisciculture de Pont Kerlo (station 5) le test est significatif avec une probabilité associée de $8,1 \%$, correspondant à un rapport $R_{0}$ de 0,67 (Figure 4). Pour les 4 autres (station 6 à 9 ), $R_{0}$ est nettement inférieur à 1 : entre 0,16 et 0,42 suivant la station, avec des probabilités associées inférieures à $0,4 \%$. On observe une augmentation régulière de l'abondance moyenne 
(1993-98) en juvéniles de saumon de l'année et du rapport $R_{0}$, en progressant vers l'aval depuis la station 7 jusqu'à la station 3 (de 4,8 ind $/ 5 \mathrm{mn}$ à 30,5 ind $/ 5 \mathrm{mn}$; Figure 3). Par la suite, les deux dernières stations aval montrent des abondances en tacons $0+$ plus réduites, ce qui pourrait être dû à leur position extrême sur la zone du cours principal du Scorff colonisée par le saumon atlantique.
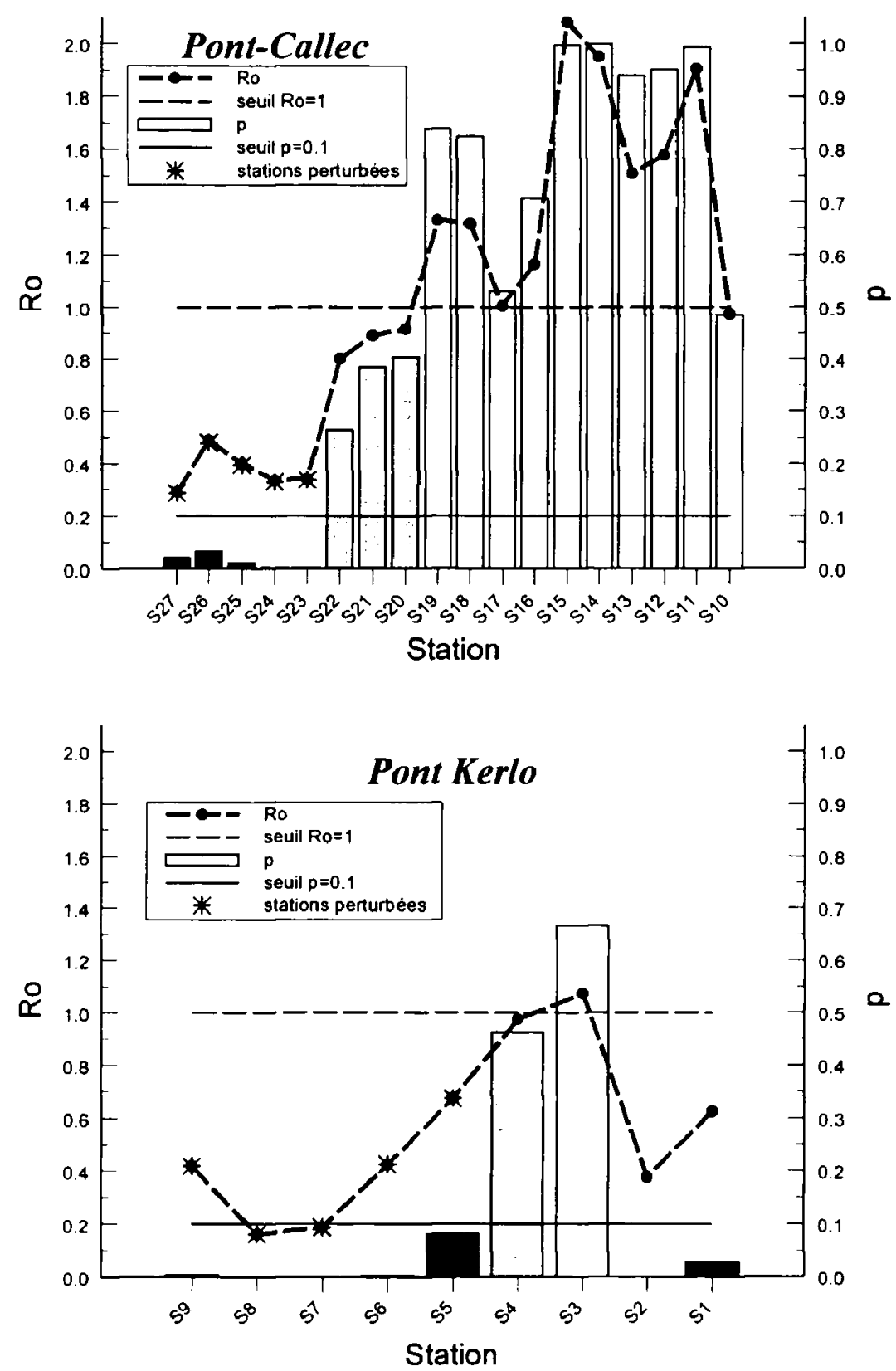

Figure 4

Déroulement de la procédure pas à pas pour la mise en évidence de l'impact des deux piscicultures (voir $\S$ Matériel et Méthodes, Traitement statistique des données).

\section{Figure 4}

Progress of the stepwise process used to reveal the impact of the two fish farms (see § Matériel et Méthodes, Traitement statistique des données). 


\section{DISCUSSION}

L'approche retenue tente de fonder sur une démarche objective la mise en évidence de l'impact de pollutions ponctuelles sur les biocénoses aquatiques en eau courante. Elle s'appuie à la fois sur des observations quantitatives distribuées le long du cours d'eau concernant une population cible (les juvéniles de saumon de l'année en l'occurrence) et sur une méthodologie de traitement de ces informations utilisant des critères d'identification des sites perturbés clairement définis. Les résultats obtenus par application de cette approche au cas des piscicultures du Scorff révèlent que, globalement sur les six années d'étude (1993-98), les abondances de juvéniles de saumon de l'année sont déprimées sur plusieurs kilomètres à l'aval des points de rejets des effluents de ces installations. Cet élément confirme le constat fait par d'autres auteurs de l'altération de différents compartiments biotiques (invertébrés, macrophytes aquatiques, poissons) d'écosystèmes lotiques par la pollution des piscicultures (BOURGET-RIVOALLAN, 1982 ; KENDRA, 1991 ; OBERDORFF et PORCHER, 1994 ; DANIEL et HAURY, 1995).

La distance à l'aval de chaque installation recouvrant les cinq stations, sur lesquelles l'abondance des tacons est significativement déprimée, constitue une valeur minimale pour l'étendue de la zone où la production de juvéniles de saumon est affectée par les rejets polluants des piscicultures. En effet, le nombre de stations identifiées comme perturbées minore le nombre de stations réellement perturbées. La méthodologie statistique utilisée pour le traitement des données est " conservatrice ", au sens où elle tend à faire plus facilement accepter qu'une station est non perturbée que l'inverse. Ceci découle de plusieurs raisons :

- tout d'abord, on a choisi de recourir à une procédure de test statistique. Or dans la problématique générale inhérente à tout test, on cherche à maîtriser l'erreur de première espèce, qui consisterait ici à déclarer que le niveau d'abondance d'une station est réduit alors que c'est faux. Ceci revient à vouloir ne rejeter qu'à " coup sûr " l'hypothèse nulle d'ordonnancement au hasard des observations d'abondance dans chaque série. On admet alors implicitement, non seulement de pouvoir conserver l'hypothèse nulle de façon erronée, mais surtout de ne pas contrôler directement la probabilité d'une telle éventualité, probabilité d'autant plus grande que le seuil de confiance du test statistique est faible (10\% dans notre cas).

- par ailleurs, le postulat initial (II), stipulant qu'au-delà d'un certain point l'impact des rejets polluants est négligeable, est contestable. On peut imaginer qu'une partie de l'effet négatif de la pollution n'est jamais résorbée, sans que l'on puisse pour autant la quantifier.

- enfin, au cours de la procédure pas à pas, on considère comme non perturbée toute station pour laquelle le résultat du test est non significatif, sans tenir compte du fait qu'elle peut être située dans un continuum de stations aux abondances réduites en aval d'effluents d'élevage (cas des stations 20 à 22 pour l'installation de Pont-Callec ; Figure 4). Or, une telle information accrédite indéniablement l'hypothèse d'une réduction d'abondance consécutive à l'impact d'une pisciculture.

Ces trois éléments, qui sont à l'origine du caractère conservateur de la méthodologie utilisée pour détecter les stations perturbées, agissent, avec des effets qui se cumulent, à deux niveaux au cours du déroulement de la démarche pas à pas :

- directement lors de chaque pas, par des erreurs de diagnostic pouvant procéder des trois causes décrites ci-dessus ;

- indirectement, lors du passage d'un pas à l'autre, par l'incorporation dans la base de référence de toute station qui n'a pas jusqu'alors été identifiée comme perturbée. Or, 
certaines de celles-ci ont pu faire l'objet d'erreurs de diagnostic, conduisant à abaisser indûment le niveau seuil en dessous duquel les abondances en tacons $0+$ seront jugées anormalement diminuées. En tendant à augmenter ainsi l'exigence d'un des critères d'identification d'une station perturbée, on augmente également le risque de considérer, à tort, une station comme non perturbée.

Le statut des stations (perturbé vs non perturbé) est déterminé à partir des six années d'étude utilisées concurremment, chacune contribuant à l'analyse sous la forme d'une série d'observations. La procédure de test de RAGO (1993), en permettant un traitement conjoint de séries multiples, s'intéresse à la détection d'un effet global (moyen) sur l'ensemble des séries. Ainsi, les résultats présentés précédemment mettent bien en évidence un impact des piscicultures sur la production de juvéniles de saumon de l'année, mais ce dernier doit être entendu au sens d'une altération appréciée en moyenne interannuelle sur la période d'étude (1993-98). Pour compléter ce point de vue global dans le temps, on peut chercher à évaluer dans quelle mesure chaque année contribue à l'effet moyen détecté. Une observation directe des profils d'abondance annuels en fonction de la distance à l'estuaire, lissés au moyen de la méthode Lowess (CLEVELAND, 1979) pour en faciliter la lecture, permet très simplement d'aborder cette question. Pour chaque série annuelle, deux lissages ont été réalisés (Figure 5) :

- l'un prend en compte l'ensemble des stations et intègre donc les perturbations induites par les rejets polluants des piscicultures;

- l'autre est établi uniquement sur les stations identifiées comme non perturbées et donne une image d'un profil d'abondance potentielle en l'absence de pollution par les piscicultures.

L'examen des données brutes et la comparaison des profils lissés révèlent :

- une tendance systématique à la réduction des abondances en tacons $0+$ sous la pisciculture de Pont Kerlo, particulièrement nette les deux années à forte abondance (1996 et 1997);

- une situation plus contrastée pour l'installation de Pont-Callec, avec à nouveau un effet marqué en 1996 et 1997, alors qu'il est absent ou peu visible les années où les abondances sont faibles à moyennes (1993-95, 1998).

Ces éléments font ressortir que l'augmentation du niveau moyen annuel des abondances, accentue le contraste entre stations perturbées et non perturbées et rend ainsi plus net l'effet de réduction des abondances de juvéniles de saumon par les rejets polluants. La procédure de test utilisée (RAGO, 1993), qui travaille à partir d'une statistique de rapport de sommes de moyennes annuelles $\left(R_{0}\right)$, est sensible à ces variations de contrastes. Les deux années à abondance élevée (1996 et 1997) contribuent donc plus fortement que les autres à l'impact moyen observé pour les deux piscicultures. L'examen des séries annuelles souligne également que l'impact de chacune des deux installations s'exprime différemment dans le temps. Ceci pourrait être le résultat d'une interaction entre des pratiques d'élevage propres à chaque installation et des contextes écologiques distincts (cf. § Site d'étude).

Pour chacune des deux piscicultures, la première station à l'amont présente une abondance moyenne faible (station $10: 16,5 \mathrm{ind} / 5 \mathrm{mn}$, station $28: 11,3 \mathrm{ind} / 5 \mathrm{mn}$ ), inférieure au niveau général des autres stations amont de la base de référence concernée et du même ordre que sur les moins touchées des stations perturbées en aval (station 5 : $16,2 \mathrm{ind} / 5 \mathrm{mn}$, station $26: 11,3 \mathrm{ind} / 5 \mathrm{mn}$; Figure 3). Aucune particularité locale identifiable 

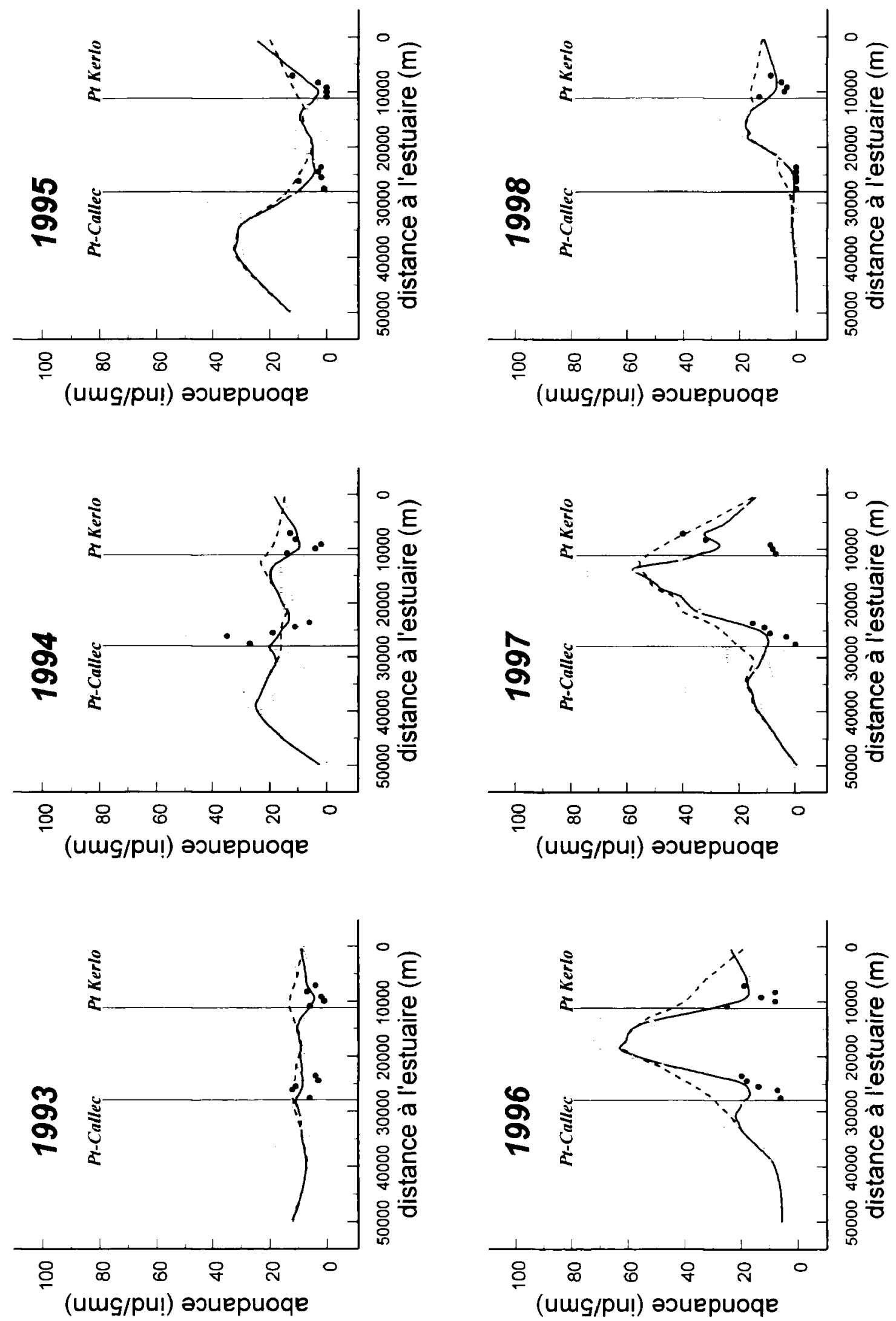


\section{Figure 5}

Profils d'abondance en tacons $0+$ le long du cours principal du Scorff en fonction de la distance à l'estuaire par année (1993-1998). Les courbes lissées sont obtenues par la méthode Lowess (largeur de la fenêtre: 12 points) en considérant toutes les stations (trait continu) ou seulement les stations non perturbées (tirets).

\section{Figure 5}

O+ parr abundance profiles along the mainstream of the Scorff $R$. according to the distance from the estuary by year (1993-1998). The smoothed curves are obtained by the Lowess's method (12 points in the smoothing window) considering all the stations (solid line) or only the non perturbed stations (dashed line).

in situ ne permet d'expliquer les abondances réduites observées sur ces stations. S'il est incontestable qu'elles ne peuvent avoir subi l'influence des rejets polluants des piscicultures, elles semblent pourtant représenter le prolongement amont d'un continuum de stations affectées par chacune des installations. La propagation d'un effet des piscicultures vers l'amont pourrait se faire par le biais des barrages qui leurs sont associés. Ces derniers, qui sont des entraves à la libre circulation des géniteurs vers l'amont, pourraient agir comme des "filtres », ne laissant progresser vers l'amont que des adultes en phase de migration active. Ces poissons, une fois l'obstacle du barrage franchi, seraient alors enclins à poursuivre leur remontée au-delà des premières zones courantes à l'amont. Les faibles abondances en tacons $0+$ constatées sur les stations correspondantes pourraient donc résulter d'une carence en géniteurs sur ces sites. L'impact des barrages des piscicultures pourrait même être plus global et influer sur la distribution des adultes au moment de la reproduction à l'échelle du cours d'eau tout entier, en induisant une relative concentration des géniteurs vers l'aval. Ces hypothèses soulignent que la seule analyse sous l'angle de l'effet des rejets polluants, pour nécessaire qu'elle soit, ne donne probablement qu'une vision partielle de l'impact écologique global d'une pisciculture.

L'effet des rejets polluants des piscicultures sur les abondances de juvéniles de saumon constaté dans cet article est corroboré par d'autres observations faites sur les mêmes sites. DANIEL et HAURY (1995) enregistrent en effet des modifications d'abondance et de composition des communautés végétales aquatiques à l'aval des deux installations étudiées ; BOURGET-RIVOALLAN (1982) constate également des altérations des peuplements d'invertébrés benthiques en aval de celle de Pont-Callec. Dans les deux cas, ces changements peuvent être reliés avec le degré de tolérance de certaines espèces vis-à-vis de traits caractéristiques de la pollution, mise en évidence de façon concomitante par ces auteurs grâce à des mesures sur la qualité de l'eau. Sur le plan des populations de poissons, BEZ (1990) note une accumulation de biomasse d'anguilles à l'aval direct de la pisciculture de Pont-Callec, alors que pour la truite commune BOURGET-RIVOALLAN (1982) observe, sous l'influence des rejets polluants, une diminution des densités accompagnée d'une augmentation de taille et de l'apparition de tissus adipeux périviscéraux chez certains individus. Des informations complémentaires sont également disponibles pour le saumon atlantique:

- des expériences in situ visant à quantifier les taux de survie lors des phases de développement embryo-larvaire sous graviers ont été menées sur les stations 9 et 11 (Figure 2), de part et d'autre de la pisciculture de Pont Kerlo (CLAUDE, 1996 ; PRÉVOST et BARDONNET, données non publiées). Pour les trois années d'observations actuellement disponibles (travail en cours), les taux de survie de l'œuf fécondé à l'alevin émergent sur la station à l'aval immédiat de la pisciculture sont nuls pour deux années et toujours nettement inférieurs à ceux observés sur la station 11 à l'amont de l'installation de Pont Kerlo. 


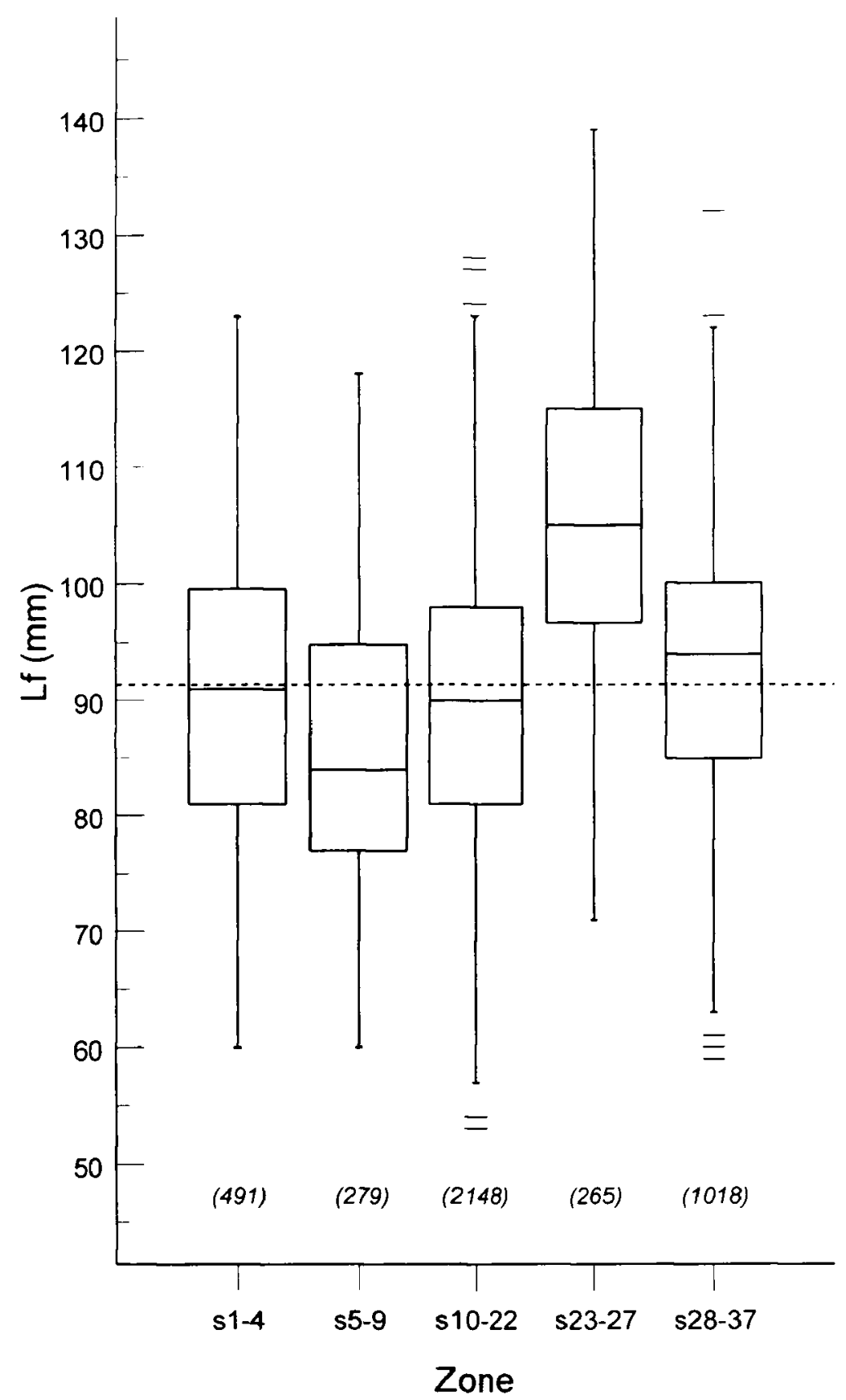

Figure 6

Boîtes de dispersion pour la taille (longueur fourche) des tacons $0+$ en fonction de la zone du cours principal du Scorff. Les échantillons sont cumulés sur la période 1993-1998 et leurs effectifs sont indiqués entre parenthèses. La moyenne générale est représentée par une ligne pointillée. Les stations 5 à 9 sont celles perturbées par la pisciculture de Pont Kerlo alors que les stations 23 à 27 le sont par celle de Pont-Callec.

Figure 6

Size (fork length) of the $0+$ parr box plots according to the zone of the Scorff $\mathbf{R}$. mainstream. Samples are pooled over the 1993-1998 period and their sizes are indicated into brackets. The overall mean is represented as a dotted line. The stations 5 to 9 are the ones perturbed by the Pont Kerlo fish farm while the stations 23 to 27 are impacted by the Pont-Callec facility. 
- les tailles des juvéniles de saumon de l'année ont été comparées entre cinq zones correspondant aux stations perturbées par chacune des deux piscicultures (stations 5 à 9 et 23 à 27) et aux trois groupes de stations contiguës ayant servi à la constitution des bases de référence utilisées pour identifier les stations perturbées (stations 1 à 4,10 à 22 et 28 à 37 ; Figure 3). La comparaison des échantillons cumulés sur les six années d'étude selon la zone révèle que sur celle perturbée par la pisciculture de Pont Kerlo la taille des juvéniles de saumon de l'année est plus faible que sur les zones adjacentes, alors que l'effet inverse est observé pour la zone sous l'influence des rejets polluants de l'installation de Pont-Callec (Figure 6). Un simple test de permutation aléatoire tenant compte de la stratification de l'échantillonnage par année (MEHTA et PATEL, 1997), appliqué à la comparaison des zones deux à deux, montre que la taille moyenne des tacons $0+$ est significativement différente $(p<0,0001)$ sur les zones perturbées par rapport aux zones adjacentes, alors qu'aucune différence significative n'est observée $(p>0,1)$ entre les zones déduites des bases de référence. Ces différences de taille peuvent être interprétées comme une altération de la croissance des individus sous l'influence de la pollution. Cette dernière pourrait avoir simultanément un effet défavorable du fait de conditions stressantes pour les poissons (par exemple, ajustement à des fluctuations de la qualité de l'eau, lutte contre des agents pathogènes) et un effet favorable du fait d'un enrichissement global du milieu (eutrophisation). L'équilibre entre ces deux effets contradictoires serait donc différent suivant la pisciculture. Ceci vient renforcer l'hypothèse, émise précédemment à partir des variations annuelles des réductions d'abondance en aval des piscicultures (Figure 5), d'une expression différente de l'impact de chacune des installations, résultant de pratiques zootechniques et de contextes écologiques eux-mêmes différents.

Le présent article met clairement en évidence l'effet négatif de la pollution engendrée par l'aquaculture intensive sur la population de saumon atlantique d'un cours d'eau. Quelles que soient les variations dans la nature de l'impact suivant l'installation, l'ensemble des informations complémentaires présentées montre que la réduction des abondances en tacons $0+$ est un élément, pouvant servir de révélateur, d'une altération globale du fonctionnement de l'écosystème à l'aval des effluents de piscicultures. Une suite logique à ce travail serait de quantifier les pertes de production de juvéniles associées et de les comparer avec la production totale du système étudié. Pour cela, il faut procéder à la fois à une estimation de la production de juvéniles de saumon en présence des piscicultures et à une évaluation du potentiel de production en l'absence de ces installations polluantes. On peut imaginer d'utiliser les données d'abondance existantes en traitant séparément le jeu de données complet, qui intègre les perturbations des piscicultures, et un jeu de données restreint, sans les stations perturbées, pour aborder la question du potentiel de production en l'absence de pollution d'origine aquacole. Les données d'abondance étant vraisemblablement structurées spatialement, une approche faisant appel aux méthodes de la géostatistique serait certainement à considérer.

\section{REMERCIEMENTS}

Les travaux présentés dans cet article ont fait l'objet d'une convention INRAJCSP $n^{\circ} 97594$. L'auteur tient à remercier Nicolas Jeannot, François Burban et Frédéric Marchand pour leur collaboration technique. Les commentaires de Jean-Luc Baglinière ont contribué à améliorer la qualité du manuscrit.

\section{BIBLIOGRAPHIE}

BAGLINIĖRE J.L., CHAMPIGNEULLE A., 1986. Population estimates of juvenile Atjantic salmon, Salmo salar, as indices of smolt production in the R. Scorff, Brittany. J. Fish Biol., 29, 467-482. 
BEZ N., 1990. La population d'anguille européenne (Anguilla anguilla) du réseau hydrographique du Scortf (Morbihan) de 1972 à 1981 - Répartition des densités et composition en taille. Mémoire D.A.A. opt. Halieutique, E.N.S.A. Rennes, I.N.R.A. Labo. écol. hydrobio., $40 \mathrm{p}$.

BOURGET-RIVOALLAN S., 1982. L'impact des piscicultures sur les rivières à salmonidés. 1 - Caractérisation générale des rejets sur le milieu. Propositions de solutions. (essai bibliographique). II - Analyse d'un exemple dans le cas du Scorff, rivière de Bretagne-sud. Mémoire D.A.A. opt. Protec. amgt. mil. nat., E.N.S.A. Rennes, I.N.R.A. Lab. écol. hydrobio., 29 p.

CLAUDE A., 1996. Le recrutement chez le saumon atlantique (Salmo salar L.) dans le Massif Armoricain. Quantification des surfaces d'habitat favorables aux juvéniles et estimation de la survie embryo-larvaire sur le Scorff (Morbihan) et l'Oir, affluent de la Sélune (Manche). Mémoire C.E.S.A. opt. Halieutique, E.N.S.A. Rennes, I.N.R.A. Labo. écol. aquat., $44 \mathrm{p}$.

CLEVELAND W.S., 1979. Robust locally weighted regression and smoothing scatterplots. J. Am. Stat. Assoc., 74, 829-836.

DANIEL H., HAURY J., 1995. Effects of fish farm pollution on phytocenoses in an acidic river (the River Scorff, South Brittany, France). Acta. Bot. Gallica, 142, 639-650.

FAURÉ A., 1977. Mise au point sur la pollution engendrée par les piscicultures. Piscic. Fr., $50,33-35$

KENDRA W., 1991. Quality of salmonid hatchery effluents during a summer low-flow season. Trans. Am. Fish. Soc., 120, 43-51.

MEHTA C., PATEL N., 1997. StatXact 3 for Windows - Statistical software for exact nonparametric inference - User manual. CYTEL Software corporation, Cambridge (Massachusetts, United States), $788 \mathrm{p}$.

OBERDORFF T., PORCHER J.P., 1994. An index of biotic integrity to assess biological impacts of salmonid farm effluents on receiving waters. Aquacult., 119, 219-235.

PRÉVOST E., 1998. État du stock de saumon atlantique (Salmo salar) du Scorff (Bretagne sud, France) en 1997 : production de smolts, retours d'adultes, échappement, taux d'exploitation et de survie. Cons. scient. Moulin des Princes, INRA/CSP, $22 \mathrm{p}$.

PRÉVOST E., BAGLINIĖRE J.L., 1995. Présentation et premiers éléments de mise au point d'une méthode simple d'évaluation du recrutement en juvéniles de saumon atlantique (Salmo salar) de l'année en eau courante in GASCUEL D., DURAND J.L., FONTENEAU A., Les recherches françaises en évaluation quantitative et modélisation des ressources et des systèmes halieutiques, 39-48, Colloques et séminaires, ORSTOM éditions, Paris.

PRÉVOST E., NIHOUARN A., 1999. Relation entre indicateur d'abondance de type CPUE et estimation de densité par enlèvements successifs pour les juvéniles de saumon atlantique (Salmo salar L.) de l'année. Bull. Fr. Pêche Piscic., 352, 19-30.

RAGO P.J., 1993. Two randomization tests for estimation of regional changes in fish abundance indices: application to North Atlantic salmon. Int. Counc. Expl. Sea, Stat. Committee, C.M. 1993/D : 35, Ref. M, 23 p.

RAMADE F., 1993. Dictionnaire encyclopédique de l'écologie et des sciences de l'environnement. Ediscience international, Paris, $822 \mathrm{p}$.

THIBAULT M., 1987. Éléments de la problématique du saumon atlantique en France in THIBAULT M., BILLARD R., La restauration des rivières à saumon, 413-425, INRA, Paris.

THIBAULT M., 1996. Écohistoire du saumon atlantique en Bretagne. Agence de l'Eau Loire - Bretagne, Orléans, $165 \mathrm{p}$. 